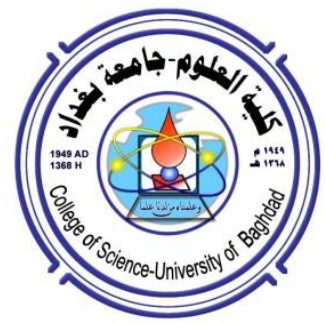

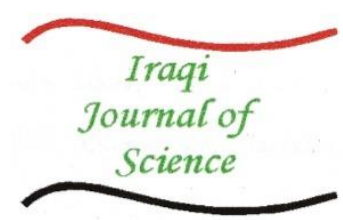

ISSN: 0067-2904

\title{
Almost Pure Ideals (Submodules) and Almost Regular Rings (Modules)
}

\author{
Imad Kh Salman, Nuhad S. Al-Mothafar
}

Department of Mathematics, College of Science, University of Baghdad, Baghdad, Iraq

\begin{abstract}
Let $\mathrm{R}$ be a commutative ring with identity and $\mathrm{M}$ be a unitary R-module. In this work we present almost pure ideal (submodule) concept as a generalization of pure ideal (submodule). Also, we generalize some properties of almost pure ideal (submodule). The study is almost regular ring (R-module).
\end{abstract}

Keyword: pure ideal, regular ring, pure submodule, regular module, almost pur submodule, almost regular module.

\section{المثاليات (المقاسات الجزئية) النقية تقرببا و الحلقات (المقاسات) لمنتظمة قريبا}

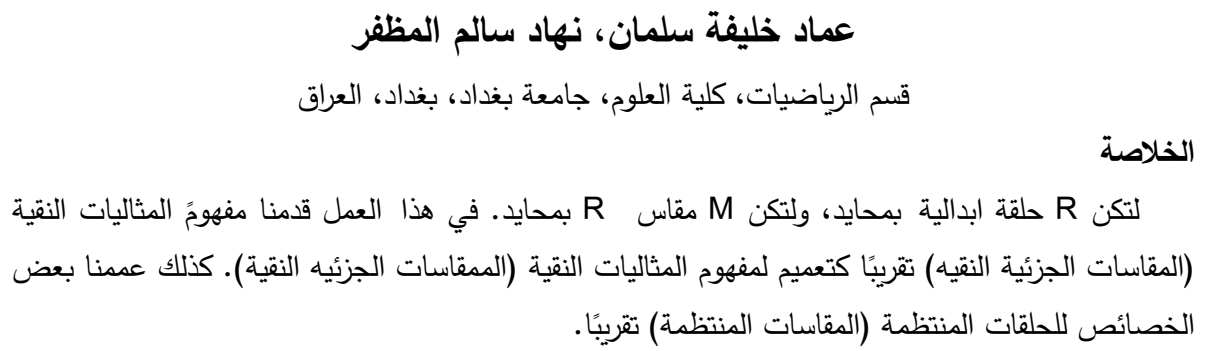

\section{Introduction}

Let $\mathrm{M}$ be a unitary $\mathrm{R}$-module and let $\mathrm{R}$ be commutative ring with identity. A submodule $\mathrm{N}$ of an R-module $\mathrm{M}$ is called pure if $\mathrm{N} \cap \mathrm{IM}=\mathrm{IN}$, for each ideal $\mathrm{I}$ of a ring $\mathrm{R}, \mathrm{E}$, Anderson and Fuller [1]. Fieldhous (1969) in [2] defined $\mathrm{N}$ to be pure in $\mathrm{M}$ if $r M \cap N=r N$ for each $r \in R$. And defined a ring $\mathrm{R}$ is regular if every ideal $\mathrm{I}$ is pure. Although Fieldhous generalize regular rings to regular modules in [2], Ware (1971) in [3] and Zelmanowliz (1972) in [4], also study regular modules. Nuhad S. Al-Mothafar and Ghaleb A. Humod in [5] study 2- pure submodules and 2- regular modules they define $\mathrm{N}$ to be 2-pure in $\mathrm{M}$ if $\mathrm{N} \cap \mathrm{I}^{2} \mathrm{M}=\mathrm{I}^{2} \mathrm{~N}$, for each ideal $\mathrm{I}$ in $\mathrm{R}$.

In this paper we introduce an almost pure ideals (submodules) concept as a generalization of pure ideals (submodules). An ideal $I$ of a ring $R$ is said to be almost pure if $I \cap J(R)=I J(R)$, where $J(R)$ is the Jacobson radical of $R$. A submodule $N$ of an R-module $M$ is called almost pure if $\mathrm{N} \cap J(R) M=J(R) N$, we study some properties of almost pure submodules. Also, we get some results of almost pure submodules like a (Prop. 3.4) let $\mathrm{M}$ be an R-module and $\mathrm{N}$ be an almost pure submodule of $\mathrm{M}$. If $\mathrm{S}$ is a submodule of $\mathrm{M}$ containing $\mathrm{N}$, then $\mathrm{N}$ is an almost pure submodule of $\mathrm{S}$.

And we present almost regular rings (modules) concepts as generalization of regular rings (modules) we generalize some properties of regular ideals (modules) to almost regular rings (modules).

This work includes three sections. In section two, we introduce almost pure ideals and almost regular rings concepts as a generalization of pure ideals and regular rings, we provided in (Prop. 2.9). If $R$ is $A$-regular integral domain such that $J(R) \neq R$, then $R$ is semisimple ring. 
In section three, we introduce almost pure submodules and almost regular modules concepts as a generalization of pure submodules and regular modules, we present (Prop. 3.16) let $\mathrm{M}$ be an almost regular R-module contain a non torsion element, then $\mathrm{R}$ is almost regular ring.

Finally in section three, we see in proposition (3.15) that if $\mathrm{M}$ is almost regular R-module, then $\forall 0 \neq x$ $\in \mathrm{M}$ such that $\operatorname{ann}(\mathrm{x}) \subseteq \mathrm{J}(\mathrm{R})$, then $\mathrm{R} / \mathrm{ann}(\mathrm{x})$ is almost regular ring.

\section{Almost Pure Ideals and Almost Regular Rings}

In this section, we introduce a generalization for pure ideals concept namely almost pure ideal. First we recall that an ideal $I$ of a ring $R$ is called pure if $I \cap J=I J$ for each ideal $J$ of $R$. It is well know that a ring $\mathrm{R}$ is (Von Neumann) regular if and only if every ideal of $\mathrm{R}$ is pure, equivalently for every $a \in R$ there exist $b \in R$ such that $a=a b a$, [2]. We start by the following definition .

\section{Definition (2.1):}

An ideal $I$ of a ring $\mathrm{R}$ is called almost pure ( for short A-pure ) ideal of $\mathrm{R}$ if $I \cap J(R)=$ $I J(R)$, where $J(R)$ is the Jacobson radical of $R$.

\section{Remarks and examples (2.2):}

1. It is clear that every pure ideal of a ring $R$ is A-pure, but the converse is not true in general, for example: In a ring Z, the ideal $\mathrm{I}=\langle 3>$ is A-pure, since $<3>\cap\{0\}=\langle 3>.\{0\}$, but $<3>$ is not pure in the ring $\mathrm{Z}$.

2. Every ideal generated by idempotent is A-pure. Since every ideal that generated by idempotent is pure [6], hence is A-pure by remark (1).

3. If $I$ and $J$ are A-pure ideals of a ring $\mathrm{R}$, but $\mathrm{I} \cap \mathrm{J}$ need not be A-pure ideal of $\mathrm{R}$. To show that if $R=Z_{4} \oplus Z_{2}$ and $I=Z_{4} \oplus 0, J=Z(\overline{1}, \overline{1})$ are A-pure ideals of R but $I \cap J=\{(\overline{0}, \overline{0}),(\overline{2}, \overline{0})\}$ is not A-pure in $\mathrm{R}$ since $(I \cap J) \cap J(R)=\{(\overline{0,} \overline{0}),(\overline{2,} \overline{0})\}$ but $(I \cap J) . J(R)=\{(\overline{0,} 0)\}$

4. It is clear that if $J(R)=\{0\}$, then every ideal in $\mathrm{R}$ is A-pure. In the ring $\mathrm{Z}$ every ideal is A-pure.

5. It is clear that $\{0\}$ and $R$ are always A-pure ideals of any ring $R$.

Recall that a ring $\mathrm{R}$ is called regular if every ideal in $\mathrm{R}$ is pure , [2]. Now, we have the following:

Definition (2.3):

A ring $R$ is called almost regular (for short A-regular) if every ideal in $R$ is A-pure. (i.e.) $I \cap J(R)$ $=\mathrm{I} J(\mathrm{R}) \quad \forall \mathrm{I} \subseteq \mathrm{R}$.

Remarks and examples (2.4):

1- Every regular ring is A-regular. But the converse is not true in general the ring $\mathrm{Z}$ is A-regular. Since $\mathrm{J}(\mathrm{Z})=0$, but not regular.

2- The rings $\mathrm{Q}, \mathrm{Z}_{\mathrm{p}}^{\infty}$, are not A-regular, since $\mathrm{J}(\mathrm{Q})=\mathrm{Q}$ and $\mathrm{J}\left(\mathrm{Z}_{\mathrm{p}}^{\infty}\right)=\mathrm{Z}_{\mathrm{p}}^{\infty}$.

Theorem (2.5):

Proof

A ring $R$ is A-regular if and only if $J(R)$ is pure ideal in $R$.

Let $I$ be an ideal of $R$. Since $R$ is A-regular, hence $I$ is A-pure in R. (i.e) $I \cap J(R)=I J(R)$. Thus $J(R)$ is pure in $R$.

The converse is clear.

Proposition (2.6)

Let $\varphi: \mathrm{R} \rightarrow \mathrm{R}^{\prime}$ be an R-epimorphism such that $\operatorname{ker} \varphi \subseteq J(R)$. If $\mathrm{R}$ is A-regular ring, then $\mathrm{R}^{\prime}$ is $\mathrm{A}$ regular ring.

Proof: Let $I^{\prime}$ be an ideal in a ring $R^{\prime}$, we have to show $I^{\prime} \cap J\left(R^{\prime}\right)=I^{\prime} J\left(R^{\prime}\right)$. Now, $I^{\prime}=\varnothing(I)$, where $I$ is an ideal in a ring $\mathrm{R}$, since $\mathrm{R}$ is A-regular ring then $I \cap J(R)=I J(R)$. Since $\varphi$ is an epimorphism whith $\operatorname{ker} \varphi \subseteq J(R)$, then $\varphi(I \cap J(R))=\varphi(I) \cap \varphi(J(R))$. Now, $\varphi(I) \cap \varphi(J(R))=\varphi(I) \cap J(R))$

$$
=\varphi(I J(R))
$$

$\varphi(I) . \varphi(J(R))$ since $\varphi$ is epimorphism and $\operatorname{ker} \varphi \subseteq J(R)$.

But $\varphi(J(R))=J\left(R^{\prime}\right)$ since $\varphi$ is epimorphism and $\operatorname{ker} \varphi \subseteq J(R)$, [7 ]. Thus $I^{\prime} \cap J\left(R^{\prime}\right)=I^{\prime} J\left(R^{\prime}\right)$.

Proposition (2.7)

Let $\mathrm{R}$ be a ring and $\mathrm{I}$ be an ideal of $\mathrm{R}$ contained in $\mathrm{J}(\mathrm{R})$. If $\mathrm{R} / \mathrm{I}$ is $\mathrm{A}$-regular ring, then $\mathrm{R}$ is $\mathrm{A}-$ regular. 


\section{Proof}

To show $R$ is A- regular ring, we have to show $K \cap J(R)=K J(R)$ for all ideal $K$ of $R$, since $R / I$ is A-regular ring and $\frac{K}{I}$ is an ideal of $\mathrm{R} / \mathrm{I}$, then $\frac{K}{I} \cap \mathrm{J}(\mathrm{R} / \mathrm{I})=\left(\frac{\mathrm{K}}{\mathrm{I}}\right)(\mathrm{J}(\mathrm{R} / \mathrm{I}))$ since $\varphi: R \rightarrow R / I$ is an epimorphism and $\operatorname{ker} \varphi \subseteq J(R)$ then $\varphi(J(R))=J(\varphi(R))$, [7 ]. hence $(\mathrm{K} / \mathrm{I}) \cap((\mathrm{J}(\mathrm{R})) / \mathrm{I})=(\mathrm{K} / \mathrm{I})$ $((\mathrm{J}(\mathrm{R})) / \mathrm{I})$, then $((\mathrm{K} \cap \mathrm{J}(\mathrm{R})) / \mathrm{I})=((\mathrm{KJ}(\mathrm{R})) / \mathrm{I})$, since $\mathrm{I} \subseteq \mathrm{J}(\mathrm{R})$. Thus $\mathrm{K} \cap \mathrm{J}(\mathrm{R})=\mathrm{KJ}(\mathrm{R})$.

Proposition (2.8):

\section{Proof}

A finite direct sum of A-regular rings $\mathrm{R}_{\mathrm{i}}$ such that $R_{\mathrm{i}} \subseteq \mathrm{J}(\mathrm{R}) \forall i=1,2, \ldots n$ is A-regular.

Let $\left\{R_{i}\right\}, \quad i=1,2, \ldots, n$ be a finite number of A-regular ring. Let $R=R_{1} \oplus R_{2} \oplus \ldots R_{n}$ be their direct sum, to show $R$ is $A$-regular. Let $n=2$, then $R=R_{1} \oplus R_{2}$, It is clear then $R / R_{1} \cong R_{2}$, since $R_{2}$ is $A$ regular by proposition(2.6) $\mathrm{R} / \mathrm{R}_{1}$ is A-regular, hence by proposition(2.7) $\mathrm{R}$ is A-regular. Using mathematical induction. Assume that the statement is true for $\mathrm{n}=\mathrm{k}$ to show that it is true for $\mathrm{k}+1$, (i.e) $\mathrm{R}=\mathrm{R}_{1} \oplus \ldots \oplus \mathrm{R}_{\mathrm{k}}$ is A-regular, and to show that $\mathrm{R}=\mathrm{R}_{1} \oplus \ldots \oplus \mathrm{R}_{\mathrm{k}} \oplus \mathrm{R}_{\mathrm{k}+1}$ is A-regular since $R / R_{1} \oplus \ldots \oplus R_{k} \cong R_{k+1}$ and $R_{k+1}$ is A-regular, then $\mathrm{R} / \mathrm{R}_{1} \oplus \ldots \oplus \mathrm{R} / \mathrm{R}_{\mathrm{k}}$ is A-regular. proposition (2.7) implies $R=R_{1} \oplus R_{2} \oplus \ldots \oplus R_{k} \oplus R_{k+1}$ is A-regular.

Recall that if $\mathrm{R}$ is integral domain then $\mathrm{R}$ is pure simple, where $\mathrm{R}$ is pure simple if it has no pure ideal exept $\{0\}$ and $R$, [8]. We know that if $R$ is regular integral domain, then $R$ is a field. But this is not true for A-regular, since $\mathrm{Z}$ is A-regular integral domain but not field.

\section{Proposition (2.9)}

If $\mathrm{R}$ is A-regular integral domain such that $\mathrm{J}(\mathrm{R}) \neq \mathrm{R}$, then $\mathrm{R}$ is semisimple ring.

proof: Since $R$ is A-regular, then by $(2.8) J(R)$ is pure ideal in $R$. But $R$ is integral domain, hence $R$ has no pure ideal exept $\{0\}$ and $R$. Since $J(R) \neq R$, hence $J(R)=0$. Thus $R$ is semisimple

Proposition (2.10)

If $\mathrm{I}$ is a prime ideal in $\mathrm{R}$ and $\mathrm{R}$ is A-regular ring such that $\mathrm{J}(\mathrm{R} / \mathrm{I}) \neq \mathrm{R} / \mathrm{I}$, then $\mathrm{R} / \mathrm{I}$ is semisimple ring.

Proof

Since I is prime ideal in R/I integral domain, and by (2.6) R/I is A-regular. Since $J(R / I) \neq R / I$, then by proposition (2.9) $\mathrm{R} / \mathrm{I}$ is semisiple.

\section{Almost Pure Submodules and Almost Regular Modules}

Recall that a sub module $\mathrm{N}$ of an R-module $\mathrm{M}$ is called pure if $\mathrm{N} \cap \mathrm{IM}=\mathrm{IN}$, for each ideal $\mathrm{I}$ of a ring $\mathrm{R}$, [1]. We start this section by the following definition.

\section{Definition (3.1)}

A submodule $\mathrm{N}$ of an $\mathrm{R}$-module $\mathrm{M}$ is called almost pure, (for short A-pure), if $\mathrm{N} \cap \mathrm{J}(\mathrm{R}) \mathrm{M}=\mathrm{J}(\mathrm{R})$ $\mathrm{N}$, where $\mathrm{J}(\mathrm{R})$ is the Jacobson radical of a ring $\mathrm{R}$.

\section{Remarks and Examples (3.2)}

1. It 's clear that every pure submodule is A-pure, but the converse is not true in general. For example the submodule $\{\overline{0}, \overline{2}\}$ in $Z_{4}$ as $Z$-module is A-pure, since $\{\overline{0}, \overline{2}\} \cap \mathrm{J}(\mathrm{Z}) \mathrm{Z}_{4}=\mathrm{J}(\mathrm{Z})\{\overline{0}, \overline{2}\}\{\overline{0}$ $, \overline{2}\} \cap\{0\} Z_{4}=\{0\} .\{\overline{0}, \overline{2}\}=\{\overline{0}\}$. But $\{\overline{0}, \overline{2}\}$ is not pure in $Z_{4}$ as $Z$-module.

2. If $\mathrm{N}$ and $\mathrm{K}$ are A-pure sub modules in an R-module $\mathrm{M}$, then $\mathrm{N} \cap \mathrm{K}$ need not be A-pure, as the following example show. If $\mathrm{M}=\mathrm{Z}_{4} \oplus \mathrm{Z}_{2}$ as $\mathrm{Z}_{4}$-module and $\mathrm{A}=\mathrm{Z}_{4} \oplus 0, \mathrm{~B}=Z_{4}(1,1)$ are A-pure submodules of $M$ but $A \cap B=\{(0,0),(2,0)\}$ is not $A$-pure in $M$. Since $(2,0) \in(A \cap B) \cap J\left(Z_{4}\right)\left(Z_{4} \oplus Z_{2}\right)$ but $(2,0) \notin \mathrm{J}\left(\mathrm{Z}_{4}\right)(\mathrm{A} \cap \mathrm{B})$.

3. It is clear that every submodule in $\mathrm{Z}$ as $\mathrm{Z}$-module is $\mathrm{A}$-pure.

4. It s clear that every direct summand of any R-module $M$ is A-pure submodule of $M$ since every direct summand is pure summodule, hence is A-pure by $\operatorname{Remark}(1)$, but the converse is not true, for example the submodule $\{\overline{0}, \overline{3}, \overline{6}\}$ of the module $Z_{9}$ as $Z$-module is A-pure since $\{\overline{0}, \overline{3}, \overline{6}\} \cap$ $\mathrm{J}(\mathrm{Z}) \mathrm{Z}_{9}=\mathrm{J}(\mathrm{Z})\{\overline{0}, \overline{3}, \overline{6}\}$ but not direct summand.

\section{Proposition (3.3)}

Let $\mathrm{M}$ be an R-module and $\mathrm{N}$ be an A-pure submodule of $\mathrm{M}$. If $\mathrm{S}$ is A-pure in $\mathrm{N}$, then $\mathrm{S}$ is an $\mathrm{A}$ pure submodule of $\mathrm{M}$.

\section{Proof}

Since $N$ is A-pure in $M$, then $N \cap J(R) M=J(R) N$ and $S$ is A-pure in $N$, then $S \cap J(R) N=J(R) S$. Now, $S \subseteq N$, implies $J(R) S=S \cap J(R) N=S \cap(N \cap J(R) M)=(S \cap N) \cap J(R) M=S \cap J(R) M$. 


\section{Proposition (3.4)}

Let $\mathrm{M}$ be an R-module and $\mathrm{N}$ be an A-pure submodule of $\mathrm{M}$. If $\mathrm{S}$ is a submodule of $\mathrm{M}$ containing $\mathrm{N}$, then $\mathrm{N}$ is an A-pure submodule of $\mathrm{S}$.

\section{Proof}

Since $\mathrm{N}$ is A-pure submodule in $\mathrm{M}$, then $\mathrm{N} \cap \mathrm{J}(\mathrm{R}) \mathrm{M}=\mathrm{J}(\mathrm{R}) \mathrm{N}$ and since $\mathrm{N} \subseteq \mathrm{S} \subseteq \mathrm{M}$ implies $\mathrm{N} \cap \mathrm{J}(\mathrm{R}) \mathrm{S}=\mathrm{N} \cap(\mathrm{J}(\mathrm{R}) \mathrm{S} \cap \mathrm{J}(\mathrm{R}) \mathrm{M})=(\mathrm{N} \cap \mathrm{J}(\mathrm{R}) \mathrm{M}) \cap \mathrm{J}(\mathrm{R}) \mathrm{S}=\mathrm{J}(\mathrm{R}) \mathrm{N} \cap \mathrm{J}(\mathrm{R}) \mathrm{S}=\mathrm{J}(\mathrm{R}) \mathrm{N}$.

Proposition (3.5): Let $\mathrm{M}$ be an R-module and $\mathrm{N}$ be an A-pure submodule of $\mathrm{M}$. If $\mathrm{K}$ is a submodule of N, then $\frac{N}{K}$ is a A-pure submodule of $\frac{M}{K}$.

\section{Proof}

$\left(\frac{N}{K}\right) \cap \mathrm{J}(\mathrm{R})\left(\frac{M}{K}\right)=\left(\frac{N}{K}\right) \cap\left(\frac{J(R) M+K}{K}\right)$

$=\frac{\mathrm{N} \cap(\mathrm{J}(\mathrm{R}) \mathrm{M}+\mathrm{K})}{K}=\frac{(\mathrm{N} \cap \mathrm{J}(\mathrm{R}) \mathrm{M})+\mathrm{K}}{K} \quad$ by module low $=\frac{\mathrm{J}(\mathrm{R}) \mathrm{N}+\mathrm{K}}{K}=J(R)\left(\frac{\mathrm{N}}{K}\right)$.

Recall that an R-module M is called an F-regular module if every submodule of M is pure , [9]. Now, we have the following definition.

\section{Definition (3.6)}

An R-module $\mathrm{M}$ is called A-regular module if every submodule of M is A-pure.

\section{Remarks and Examples (3.7):}

1. It is clear that every F-regular is A-regular R-module but the convers is not true in general. For example $Z_{4}$ as $Z$-module is A-regular since every submodule of $Z_{4}$ is $A$-pure but $Z_{4}$ is not F-regular, see remark and examples (3.2) (1).

2. The $\mathrm{Z}$-module $\mathrm{Z}$ is A-regular since every submodules of $\mathrm{Z}$ is $\mathrm{A}$-pure. But $\mathrm{Z}$ as $\mathrm{Z}$-module is not $\mathrm{F}$ regular.

3. The $Z_{9}$ as $\mathrm{Z}$-module is A-regular since every submodule of $\mathrm{Z}_{9}$ is $\mathrm{A}$-pure. But $\mathrm{Z}_{9}$ is not F-regular since $\{\overline{0}, \overline{3}, \overline{9}\}$ is not pure, see remark and examples (3.2) (1).

4. The $\mathrm{Z}_{12}$ as $\mathrm{Z}$-module is A-regular since every submodule of $\mathrm{Z}_{12}$ is $\mathrm{A}$-pure, but $\mathrm{Z}_{12}$ is not F-regular since the submodule $\langle\overline{2}>$ is not pure.

5. $\mathrm{Q}$ as $\mathrm{Z}$-module is $\mathrm{A}$-regular since $\mathrm{Q} \cap \mathrm{J}(\mathrm{Z}) \mathrm{Q}=\mathrm{J}(\mathrm{Z}) \mathrm{Q}$, but $\mathrm{Q}$ as $\mathrm{Z}$-module is not F-regular.

\section{Theorem (3.8):}

Let $\mathrm{M}$ be an R-module. The following statements are equivalent:

1. $\mathrm{M}$ is A-regular module.

2. Every cyclic submodule of $M$ is A-pure in $M$.

3. Every finitely generated submodule of $\mathrm{M}$ is A-pure in $\mathrm{M}$.

\section{Proof}

(1) $\rightarrow$ (2) it follows by definition (3.6).

$(2) \rightarrow(1)$ Assume that every cyclic submodule of $\mathrm{M}$ is A-pure and let $\mathrm{N}$ be any submodule of $\mathrm{M}$. Let $\mathrm{x} \in \mathrm{N} \cap \mathrm{J}(\mathrm{R}) \mathrm{M}$, then $\mathrm{x} \in \mathrm{N}$ and $\mathrm{x} \in \mathrm{J}(\mathrm{R}) \mathrm{M}$ then $\mathrm{x} \in<\mathrm{x}>\cap \mathrm{J}(\mathrm{R}) \mathrm{M}=\mathrm{J}(\mathrm{R})<\mathrm{x}>\subseteq \mathrm{J}(\mathrm{R}) \mathrm{N}$.

(1) $\rightarrow$ (3). It follows by definition (3.6).

(3) $\rightarrow$ (2). It is clear.

The following proposition shows that the factor module of A-regular module is A-regular.

\section{Proposition (3.9):} of $\mathrm{M}$.

Let $\mathrm{M}$ be an R-module, then $\mathrm{M}$ is A-regular if and only if $\mathrm{M} / \mathrm{N}$ is A-regular for every submodule $\mathrm{N}$

\section{Proof}

Let $\mathrm{N}$ be a submodule of an R-module $\mathrm{M}$ and $\mathrm{K}$ be any submodule of $\mathrm{M}$ containg N. Since $\mathrm{M}$ is Aregular, then $\mathrm{K}$ is A-pure in $\mathrm{M}$, hence $\mathrm{K} / \mathrm{N}$ is A-pure in $\mathrm{M} / \mathrm{N}$ proposition (3.5). Thus $\mathrm{M} / \mathrm{N}$ is $\mathrm{A}$-regular. The converse is easily by taking $\mathrm{N}=0$.

\section{Corollary (3.10)}

Let $\mathrm{M}$ and $\mathrm{M}^{\prime}$ be $\mathrm{R}$-modules and $f: \mathrm{M} \rightarrow \mathrm{M}^{\prime}$ is an $\mathrm{R}$ - epimorphisim. If $\mathrm{M}$ is A-regular $\mathrm{R}$-module, then $\mathrm{M}^{\prime}$ is A-regular. 


\section{Proof}

Since $f: \mathrm{M} \rightarrow \mathrm{M}^{\prime}$ be an $\mathrm{R}$ - epimorphisim and $\mathrm{M}$ is A-regular $\mathrm{R}$-module, then $\mathrm{M} /$ kerf is A-regular $\mathrm{R}$ module by proposition (3.9). But $\mathrm{M} / \mathrm{kerf}$ is isomorphic to $\mathrm{M}^{\prime}$ by the first isomorphism theorem. Thus $\mathrm{M}^{\prime}$ is A-regular.

\section{Corollary (3.11)]}

Every submodule of A-regular module is A-regular module.

\section{Proof}

Let $\mathrm{N}$ be a submodule of an A-regular $\mathrm{M}$. To show that $\mathrm{N}$ is A-regular R-module, let $\mathrm{K}$ be any submodule of N. Since M is A-regular. Hence we have:

$$
\begin{array}{lr}
K \cap J(R) N=K \cap(N \cap J(R) M) & \text { since } N \text { is A-p } \\
=(K \cap N) \cap J(R) M & \\
=K \cap J(R) M & \text { since } K \text { is A-pure in } M \\
=J(R) N &
\end{array}
$$

since $\mathrm{N}$ is A-pure in $\mathrm{M}$

Therefor $\mathrm{K}$ is A-pure in $\mathrm{N}$ which implies that $\mathrm{N}$ is A-regular.

Recall that an R-module $M$ is called F-regular if and only if $\forall x \in M$ and $\forall r \in R, \exists t \in R$ such that rx=rtrx , [6].

\section{Preposition (3.12):}

If $\mathrm{M}$ is A-regular R-module, then for every nonzero element $\mathrm{x}$ in $\mathrm{M}$ and for each $\mathrm{r} \in \mathrm{J}(\mathrm{R})$, there exit $\mathrm{t} \in \mathrm{R}$ such that $\mathrm{rx}=\mathrm{rtrx}$.

\section{Proof}

Let $0 \neq x \in M$ and $r \in J(R)$.Since $r x \in J(R) M$ and $r x \in\langle r x>$ implies $r x \in J(R) M \cap<r x\rangle$ . But M is A-regular. $\langle r x\rangle \cap J(R) M=J(R)\langle r x\rangle$. Hence $r x \in J(R)\langle r x\rangle$, which implies that $r x=r t r x$ where $t \in R$

\section{Preposition (3.13):}

let $\mathrm{M}$ be an R-module is each nonzero element $\mathrm{x}$ in $\mathrm{M}$ and for every $\mathrm{r} \in \mathrm{J}(\mathrm{R}), r x=r t r x$ for some $t \in R$, then $\mathrm{M}$ is A-regular.

\section{Proof}

let $\mathrm{N}$ be a submodule of a module $\mathrm{M}$, to show $N \cap J(R) M=J(R) N$. Let $y \in N \cap J(R) M$, then $y \in N$ and $y \in J(R) M$, hence $y=r m$ where $\mathrm{r} \in \mathrm{J}(\mathrm{R})$ and $\mathrm{m} \in M, y=r m=r t r m$, for some $t \in R$. Thus $y \in J(R) N$, which implies that $\mathrm{M}$ is A-regular.

\section{corollary (3.14)}

let $\mathrm{R}$ be an A-regular ring, then for each $r \in J(R), r=r t r$ for some $t \in R$

\section{Proposition (3.15)} ring.

If $\mathrm{M}$ is A-regular R-module, then $\forall 0 \neq \mathrm{x} \in \mathrm{M}$ such that $\operatorname{ann}(\mathrm{x}) \subseteq \mathrm{J}(\mathrm{R})$, then $\mathrm{R} / \mathrm{ann}(\mathrm{x})$ is A-regular

\section{Proof}

Let $\mathrm{o} \neq \mathrm{x} \in \mathrm{M}$ and $\overline{\mathrm{r}} \in \mathrm{J}(\mathrm{R} / \mathrm{ann}(\mathrm{x}))=\varphi J(R))$ where $\varphi: R \rightarrow \mathrm{R} / \mathrm{ann}(\mathrm{x})$ is an epimorphism, then ther exist $r \in J(R)$ such that $\varphi(r)=\bar{r}$. Since $M$ is A-regular R-module, $r \in J(R), \exists t \in R$ such that $r x=r t r x$, then (r-rtr) $x=0$, then $r-r t r \in a n n(x)$, hence $r+\operatorname{ann}(x)=r \operatorname{tr}+\operatorname{ann}(x)$, which implise that $\bar{r}=\bar{r} \overline{\mathrm{t}} \bar{r}$. Thus $\mathrm{R} / \mathrm{ann}(\mathrm{x})$ is A-regular ring.

Recall that $\mathrm{T}(\mathrm{M})=\{\mathrm{m} \in \mathrm{M}$ :ther exit $\mathrm{r} \in \mathrm{R}$ such that $\mathrm{r} \cdot \mathrm{m}=0\}, \mathrm{T}(\mathrm{M})=$ the set of all torsion elements. If $\mathrm{T}(\mathrm{M})=\mathrm{M}$, then $\mathrm{M}$ is called torsion R-module , [10].

\section{Proposition (3.16)}

\section{Proof}

Let $\mathrm{M}$ be an A-regular R-module contains a non torsion element, then R is A-regular ring.

Let $\mathrm{x} \in \mathrm{M}$ be a non torsion element, then $[0: \mathrm{x}]=0=\operatorname{ann}(\mathrm{x})$, so by $(3.15), \mathrm{R} / \mathrm{ann}(\mathrm{x}) \cong \mathrm{R}$ is A-regular by (3.10)

\section{References}

1. Anderson, F.W. and Fuller, K.R. 1974. Rings and Categories of Modules. Springer-Verlag.

2. Fieldhous, D.J. 1969. Pure Theories, Math.Ann.184: 1-18.

3. Ware, R. 1971. Endomorphism Rings of Projective Modules, Trans. Amer. Math. Soc. 155: 233256.

4. Zelmanowitz, J. 1972. Regular Modules, Trans. Amer. Math. Soc.163: 341-355. 
5. Al-Mothafar, N. S. and Humod, G. A. 2015. 2- Regular Modules, Ibn Al-Haitham.J.for Pure and Appl. Sci., 28(2): 184-192

6. Yaseen, S. M. 1993. On F-regular Modules, University of Baghdad (1993).

7. Kasch.F, 1982. Modulers and Rings, Acad. Press, London.

8. Fieldhous, D.J. 1976. Pure Simple and Indecomposable Rings. Can. Math. Bull. 13: 71-78

9. Brown.B, McCoy.N.H. 1950. The Maximal Regular Ideal of a Ring. Proc. Amer. Math. Soc. 1: $165-171$.

10. Rotman, J. J. 1979. An Introduction to Homological Algebra. Academic Press, New York. 